\title{
On using the WMAP distance information in constraining the time evolving equation of state of dark energy
}

\author{
Hong $\mathrm{Li}^{1}$, Jun-Qing Xia ${ }^{2}$, Gong-Bo Zhao ${ }^{3}, \mathrm{Zu}^{-H u i}$ Fan $^{1}$ \& Xinmin Zhang ${ }^{2}$
}

\begin{abstract}
Recently, the WMAP group has published their five-year data and considered the constraints on the time evolving equation of state of dark energy for the first time from the WMAP distance information. In this paper, we study the effectiveness of the usage of these distance information and find that these compressed CMB information can give similar constraints on dark energy parameters compared with the full CMB power spectrum if dark energy perturbations are included, however, once incorrectly neglecting the dark energy perturbations, the difference of the results are sizable.
\end{abstract}

Subject headings: Cosmology: theory - (Cosmology:) cosmic microwave background - (Cosmology:) cosmological parameters

\section{Introduction}

The newly released Wilkinson Microwave Anisotropy Probe five-year data (WMAP5) (Hinshaw et al. 2008; Nolta et al. 2008; Dunkley et al. 2008; Komatsu et al. 2008a), detecting the Cosmic Microwave Background (CMB) to an unprecedented precision, make it possible to improve the constraints on almost all the cosmological parameters, including the equation-of-state (EoS) $w$ of the unknown energy budget, dark energy. Defined as the ratio of pressure over energy density, $w=P / \rho$, EoS can be used to classify various dark energy models, such as quintessence (Wetterich 1995; Ratra \& Peebles 1998; Peebles \& Ratra 1998; Wetterich 1988), phantom (Caldwell 2002), quintom (Feng et al. 2005), k-essence (Armendariz-Picon et al. 2000; Armendariz-Picon et al. 2001), etc., which is of great theoretical significance to unveil the mystery of dark energy. Therefore, trying to study the

\footnotetext{
${ }^{1}$ Department of Astronomy, School of Physics, Peking University, Beijing, 100871, P. R. China.

${ }^{2}$ Institute of High Energy Physics, Chinese Academy of Science, P. O. Box 918-4, Beijing 100049, P. R. China; xiajq@mail.ihep.ac.cn.

${ }^{3}$ Department of Physics, Simon Fraser University, Burnaby, BC, V5A 1S6, Canada.
} 
evolution history of EoS of dark energy plays a crucial role in modern observational cosmology (Huterer \& Starkman 2003; Wang \& Tegmark 2005; Zhao et al. 2007c). Simply put, one can choose an arbitrary parametrization of $w$ and constrain the introduced dark energy parameters from the astronomical observational data, including CMB, Supernova type Ia (SN Ia), Large Scale Structure (LSS) and so forth (Wang \& Mukherjee 2007; Wright 2007; Zhao et al. 2007a; Zhao et al. 2007b).

Recently, the WMAP group has released their five-year data and for the first time considered the constraints on the time evolving EoS of dark energy using the WMAP distance information. This method has the advantage of reducing computation time by orders of magnitude, yet the effectiveness and the level of approximation compared with the full CMB power spectrum computation remain unclear. In this paper, we make a thorough test of this simplified method to investigate whether it is safe to constrain dark energy with time evolving EoS. Our paper is structured as follows: In Section II we describe the method and the data; in Section III we present our main results; finally we present our conclusions in Section IV.

\section{Method and data}

To study the dynamical behavior of dark energy, we choose the parametrization of the time evolving EoS of dark energy given by (Chevallier \& Polarski 2001; Linder 2003; Komatsu et al. 2008a):

$$
w(a)=w_{0}+w_{a}(1-a),
$$

where $a=1 /(1+z)$ is the scale factor and $w_{a}=-d w / d a$ characterizes the "running" of the EoS (RunW henceforth). For the $\Lambda$ CDM model, $w_{0}=-1$ and $w_{a}=0$.

When using the MCMC global fitting strategy to constrain cosmological parameters, it is crucial to include dark energy perturbations, especially for the time evolving EoS of dark energy models. This issue has been realized by many researchers including the WMAP group (Weller \& Lewis 2003; Yeche et al. 2006; Zhao et al. 2005; Xia et al. 2006; Spergel et al. 2007). However, when the parameterized EoS crosses -1, one cannot handle the dark energy perturbations based on quintessence, phantom, k-essence and other non-crossing models. By virtue of quintom, the perturbations at the crossing points are continuous, thus we have proposed a technique to treat dark energy perturbations in the whole parameter space. For details of this method, we refer the readers to our previous papers (Zhao et al. 2005; Xia et al. 2006).

In this study, we have modified the publicly available Markov Chain Monte Carlo pack- 
age CosmoMC1 (Lewis \& Bridle 2002) to include the dark energy perturbations with EoS across -1 . Furthermore, we assume purely adiabatic initial conditions and a flat universe. Our most general parameter space is:

$$
\mathbf{P} \equiv\left(\omega_{b}, \omega_{c}, \Theta_{s}, \tau, w_{0}, w_{a}, n_{s}, \ln \left(10^{10} A_{s}\right)\right),
$$

where $\omega_{b} \equiv \Omega_{b} h^{2}$ and $\omega_{c} \equiv \Omega_{c} h^{2}$, where $\Omega_{b}$ and $\Omega_{c}$ are the baryon and cold dark matter densities relative to the critical density, $\Theta_{s}$ is the ratio (multiplied by 100) of the sound horizon at decoupling to the angular diameter distance to the last scattering surface, $\tau$ is the optical depth to reionization, $A_{s}$ and $n_{s}$ are the amplitude and the tilt of the power spectrum of primordial scalar perturbations. For the pivot scale of the primordial spectrum we set $k_{*}=0.05 \mathrm{Mpc}^{-1}$.

The WMAP distance information used by WMAP group include the "shift parameter" $R$, the "acoustic scale" $l_{A}$ and the photon decoupling epoch $z_{*} . R$ and $l_{A}$ correspond to the ratio of angular diameter distance to the decoupling era over Hubble horizon and sound horizon at decoupling respectively, given by

$$
\begin{aligned}
& R\left(z_{*}\right)=\sqrt{\Omega_{m} H_{0}^{2}} \chi\left(z_{*}\right), \\
& l_{A}\left(z_{*}\right)=\pi \chi\left(z_{*}\right) / \chi_{s}\left(z_{*}\right),
\end{aligned}
$$

where $\chi\left(z_{*}\right)$ and $\chi_{s}\left(z_{*}\right)$ denote the comoving distance to $z_{*}$ and comoving sound horizon at $z_{*}$ respectively. The decoupling epoch $z_{*}$ is given by (Eisenstein \& Hu 1998)

$$
z_{*}=1048\left[1+0.00124\left(\Omega_{b} h^{2}\right)^{-0.738}\right]\left[1+g_{1}\left(\Omega_{m} h^{2}\right)^{g_{2}}\right],
$$

where

$$
g_{1}=\frac{0.0783\left(\Omega_{b} h^{2}\right)^{-0.238}}{1+39.5\left(\Omega_{b} h^{2}\right)^{0.763}}, g_{2}=\frac{0.560}{1+21.1\left(\Omega_{b} h^{2}\right)^{1.81}} .
$$

The WMAP distance information encode in part of the CMB information and can constrain cosmological parameters to some extent. It is worth carefully investigating on the effectiveness of the constraints from the distance information compared with the full CMB power spectrum computation. To do this, we follow the procedure shown in the flow chart:

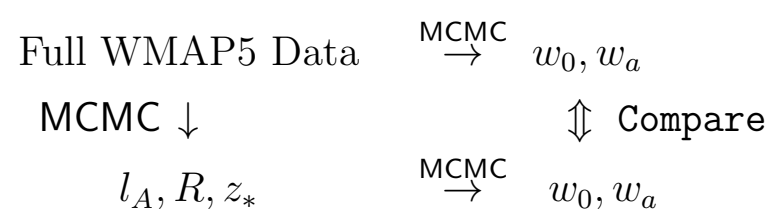

\footnotetext{
${ }^{1}$ http://cosmologist.info/cosmomc/.
} 
which are detailed as follows:

1. Making a global fitting with MCMC method to constrain $w_{0}, w_{a}$, and also $l_{A}, R$ and $z_{*}$ using the full WMAP5 power spectrum. In this step, we have done two types of calculations, one with and the other without dark energy perturbations;

2. Using the resultant $l_{A}, R$ and $z_{*}$ to constrain dark energy parameters $w_{0}$ and $w_{a}$;

3. Comparing the results of the constraints on $w_{0}$ and $w_{a}$ obtained from step 1 and 2 .

In step 1, we calculate the likelihood of CMB power spectrum using the routine supplied by the WMAP group2. In step 2, we calculate the likelihood of WMAP distance information as follows (Komatsu et al. 2008a):

$$
\chi^{2} \equiv-2 \ln L=\left(x_{i}^{t h}-x_{i}^{d a t a}\right)\left(C^{-1}\right)_{i j}\left(x_{j}^{t h}-x_{j}^{d a t a}\right),
$$

where $x=\left(l_{A}, R, z_{*}\right)$ is the parameter vector and $\left(C^{-1}\right)_{i j}$ is the inverse covariance matrix for the WMAP distance information.

Since the purpose of this paper is not to make a global analysis, in order to see the effects of the other cosmological data, we include the gold sample of 182 SN Ia (Riess et al. 2007) for a joint constraint on EoS of dark energy in the combination with the WMAP5 data. In this study we also make use of Hubble Space Telescope (HST) measurement of the Hubble parameter $H_{0} \equiv 100 h \mathrm{~km} \mathrm{~s}^{-1} \mathrm{Mpc}^{-1}$ by multiplying the likelihood by a Gaussian likelihood function centered around $h=0.72$ and with a standard deviation $\sigma=0.08$ (Freedman et al. 2001), and a Gaussian prior on the baryon density $\Omega_{b} h^{2}=0.022 \pm 0.002(1 \sigma)$ from Big Bang Nucleosynthesis (BBN) (Burles et al. 2001).

\section{Results}

The WMAP distance information are extracted from the full WMAP5 power spectrum by assuming a certain cosmological model, and they should be model dependent. In Fig 11we present the one dimensional distributions of the WMAP distance information for different cosmological models.

In the upper three panels of Fig.1 we show the distributions of $l_{A}, R$ and $z_{*}$ for five cosmological models: flat $\Lambda \mathrm{CDM}$ model; $\Lambda \mathrm{CDM}$ with curvature; flat $\Lambda \mathrm{CDM}$ model with

\footnotetext{
${ }^{2}$ Legacy Archive for Microwave Background Data Analysis (LAMBDA), http://lambda.gsfc.nasa.gov/.
} 
massive neutrinos, with running of spectral index and with tensor perturbations, respectively. We find that the distributions of $R$ and $z_{*}$ are quite different in these five cases, while the acoustic scale $l_{A}$ does not change significantly. These results indicate that when using these distance information to constrain cosmological parameters, one should be clear about the assumed cosmological model. In Table I we also list the median $1 \sigma$ constraints on the WMAP distance information from the full WMAP5 data for different cosmological models.
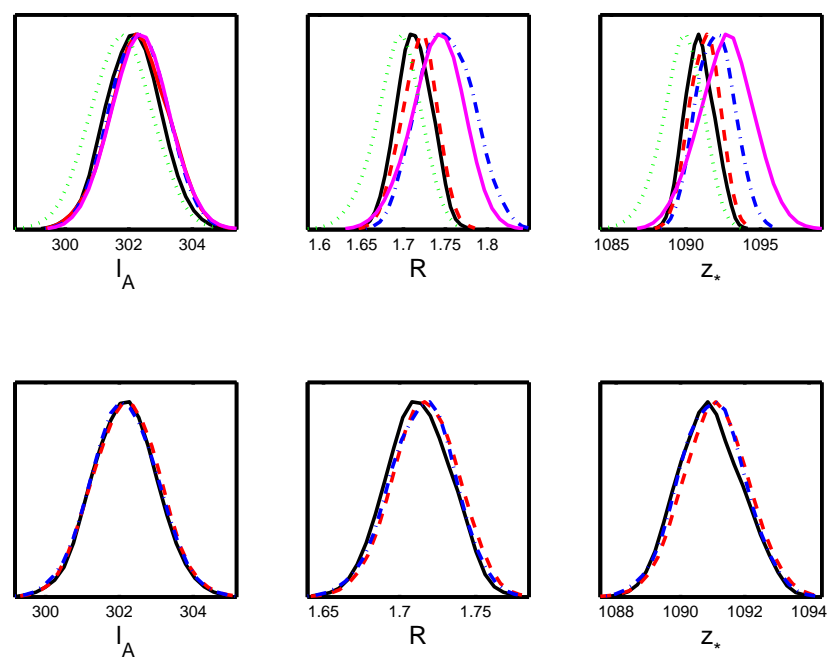

Fig. 1.- One dimensional posterior distributions of $l_{A}, R$ and $z_{*}$ with the WMAP5 data for different cosmological models. In the upper panels, the black solid line is given by the standard flat $\Lambda \mathrm{CDM}$ model, while the red dashed line, the blue dash-dotted line, purple solid line and the green dotted line are given by $\Lambda$ CDM with non-zero $\Omega_{k}$, flat $\Lambda$ CDM with $f_{\nu}$, with $\alpha_{s}$ and with $r$ respectively. In the lower panels, the black solid line is still from the standard $\Lambda \mathrm{CDM}$ model, while the red dashed line and the blue dash-dotted lines are given by the dark energy model with time evolving EoS (RunW model) with and (incorrectly) without dark energy perturbations respectively.

In the lower three panels of Fig.1 we show the results for three flat models with different dark energy properties: $\Lambda$ CDM model, RunW model with and (incorrectly) without dark energy perturbations. These results do not show significant differences in the WMAP distance information among different dark energy models. We also compare the results obtained with and (incorrectly) without dark energy perturbations and find that simply switching off dark energy perturbations does not bias the results much at this stage 3 . In the following

\footnotetext{
${ }^{3}$ The distance information are determined by the background parameters and not affected by dark energy perturbations significantly.
} 
calculations, we use the WMAP distance information obtained from the RunW model with dark energy perturbations included. The corresponding inverse covariance matrix is shown in Table II.

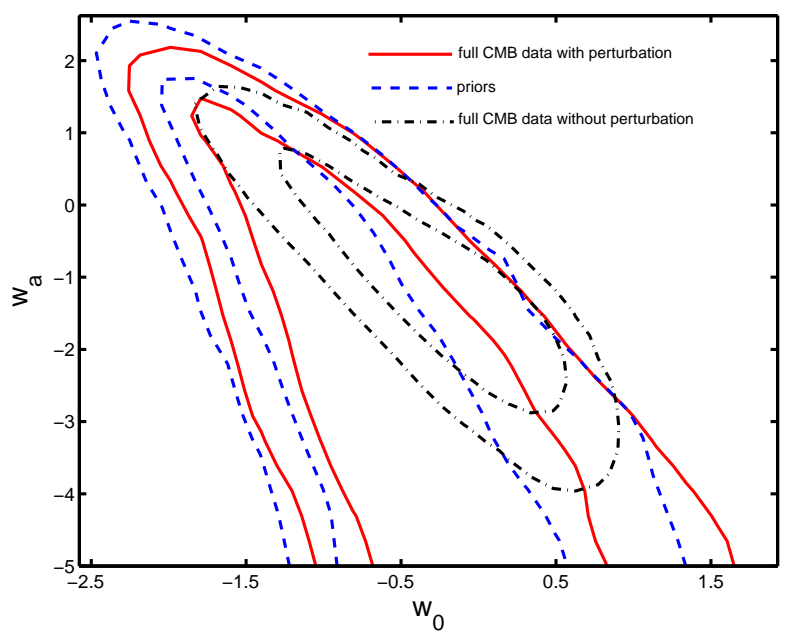

Fig. 2.- $68 \%$ and $95 \%$ confidence levels constraints on $\left(w_{0}, w_{a}\right)$ from full WMAP5 data and WMAP distance information respectively. Red solid lines are obtained from the full WMAP5 data including dark energy perturbations; black dash-dotted lines are from the full WMAP5 data incorrectly neglecting dark energy perturbations; and blue dashed lines are from WMAP distance information.

In Fig, 2 we compare the constraints on $w_{0}$ and $w_{a}$ obtained from the full WMAP5 power spectrum with the one obtained from WMAP distance information given in Table I and II. From this plot we can see that the WMAP distance information and the full WMAP5 power spectrum with dark energy perturbations included can give quite similar constraints on $w_{0}$

Table I. Median $1 \sigma$ constraints on WMAP distance information using full WMAP5 data for different cosmological models.

\begin{tabular}{c|c|c|c}
\hline \hline Models & $l_{A}$ & $R$ & $z_{*}$ \\
\hline$\Lambda \mathrm{CDM}$ & $302.15 \pm 0.842$ & $1.71 \pm 0.021$ & $1090.92 \pm 0.969$ \\
\hline$\Lambda \mathrm{CDM}+\Omega_{K}$ & $302.32 \pm 0.899$ & $1.72 \pm 0.021$ & $1091.26 \pm 1.004$ \\
\hline$\Lambda \mathrm{CDM}+m_{\nu}$ & $302.30 \pm 0.873$ & $1.75 \pm 0.031$ & $1091.98 \pm 1.244$ \\
\hline$\Lambda \mathrm{CDM}+\alpha_{s}$ & $302.36 \pm 0.878$ & $1.74 \pm 0.031$ & $1092.72 \pm 1.817$ \\
\hline$\Lambda \mathrm{CDM}+r$ & $301.76 \pm 0.944$ & $1.69 \pm 0.027$ & $1089.72 \pm 1.366$ \\
\hline RunW with pert. & $302.20 \pm 0.865$ & $1.72 \pm 0.021$ & $1091.10 \pm 0.991$ \\
\hline RunW w/o pert. & $302.14 \pm 0.875$ & $1.71 \pm 0.021$ & $1090.97 \pm 0.985$ \\
\hline \hline
\end{tabular}


and $w_{a}$. However, when the dark energy perturbations are incorrectly switched off (black dash-dotted lines in Fig.2), the results between the two methods are quite different.

The WMAP distance information mainly include the information of the oscillatory structures of the CMB power spectrum, which come from the small angular scale (large $l$ ) of the power spectrum. On the other hand, for the full CMB power spectrum, they combine more information than the distance information, especially at large angular scale (small $l$ ). At large angular scale, they are affected by the late Integrated Sachs-Wolfe (ISW) effects, which are dark energy dependent. Thus tighter constraints on $\left(w_{0}, w_{a}\right)$ are anticipated by using the full CMB spectrum than those from using the distance priors only. This is clearly demonstrated in Fig 2 (dashed contours versus dash dotted one). It is noted that the dash dotted contours are calculated without including dark energy perturbations, and thus the constraining power of the late ISW effect on dark energy parameters is fully realized. However, when including the dark energy perturbations, which are mainly effective at small $l$, the constraints on dark energy parameters from the late ISW effects are significantly reduced, resulting in similar contours shown by the dashed and solid lines in Fig:24 (Komatsu 2008b). The differences between the solid and the dash dotted contours also show that how biased results can be obtained if the dark energy perturbations are incorrectly neglected in the full CMB data analysis (Xia et al. 2006; Spergel et al. 2007).

In Fig 3, we give the constraints on dark energy parameters by adding the SN Ia data. We can see that the constraints on dark energy parameters are tightened and the differences between the results obtained from "full WMAP5 power spectrum + SN Ia" and from "WMAP distance information + SN Ia" become insignificant.

\footnotetext{
${ }^{4}$ In our analysis we use a specific parametrization the "RunW" model. We expect that our results hold qualitatively for other dark energy parameterizations. Quantitatively however, the specific results are dependent on the detailed calculations on different dark energy parameterizations that are used.
}

Table II. Inverse covariance matrix for the WMAP distance information $l_{A}, R$ and $z_{*}$ in RunW dark energy model when including dark energy perturbations.

\begin{tabular}{cccc}
\hline \hline & $l_{A}\left(z_{*}\right)$ & $R\left(z_{*}\right)$ & $z_{*}$ \\
\hline$l_{A}\left(z_{*}\right)$ & 1.795 & 31.596 & -1.146 \\
$R\left(z_{*}\right)$ & & 5409.68 & -94.58 \\
$z_{*}$ & & & 2.891 \\
\hline \hline
\end{tabular}




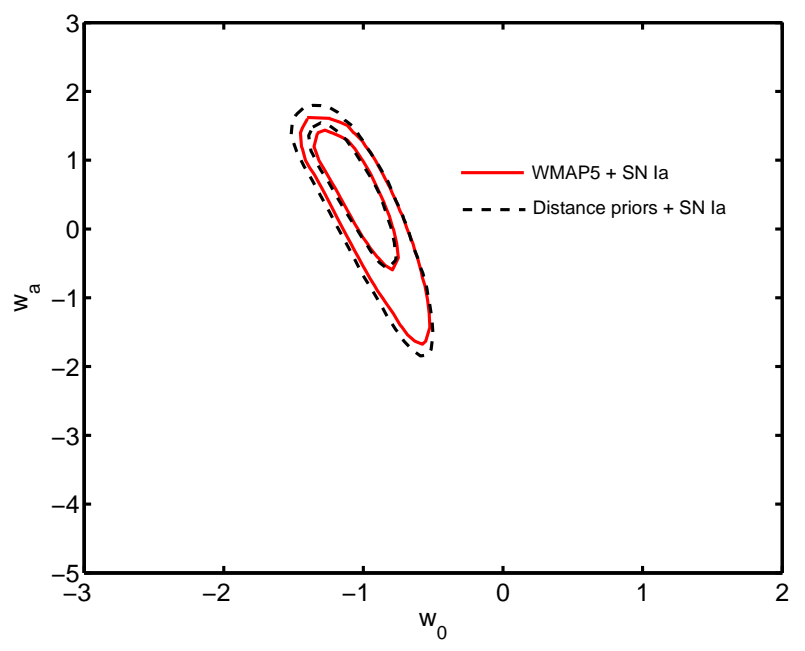

Fig. 3.- Two-dimensional constraints on the parameters of dark energy from the combined WMAP5 and SN Ia. The red solid and black dotted lines are the constraints from "full WMAP5 data + SN Ia" with dark energy perturbations and "WMAP distance information + SN Ia" respectively.

\section{Summary}

In this paper, we have studied the effectiveness of the WMAP distance information on constraining the dark energy parameters, by comparing with the full WMAP5 power spectrum analysis. We first present the level of the model dependence of the distance information in different cosmological models. We further clarify that by taking into account dark energy perturbations properly, the WMAP distances can give unbiased information on dark energy parameters relative to the full CMB analysis.

\section{Acknowledgments}

We acknowledge the use of the Legacy Archive for Microwave Background Data Analysis (LAMBDA). Support for LAMBDA is provided by the NASA Office of Space Science. We have performed our numerical analysis in the Shanghai Supercomputer Center (SSC). We would like to thank Eiichiro Komatsu, Tao-Tao Qiu and Hua-Hui Xiong for discussions. This work is supported in part by the funding support from the China postdoctoral science foundation and the National Natural Science Foundation of China under Grant Nos. 90303004, 10533010 and 10675136 and by the Chinese Academy of Science under Grant No. KJCX3-SYW-N2. GZ is supported by National Science and Engineering Research Council 
of Canada (NSERC).

\section{REFERENCES}

Armendariz-Picon, C., Mukhanov, V. \& Steinhardt, P., 2000, Phys. Rev. Lett., 85, 4438

Armendariz-Picon, C., Mukhanov, V. \& Steinhardt, P., 2001, Phys. Rev. D, 63, 103510

Burles, S., Nollett, K., \& Turner, M., 2001, ApJ, 552, L1

Caldwell, R. R., 2002, Phys. Lett. B, 54523

Chevallier, M. \& Polarski, D., 2001, Int. J. Mod. Phys. D, 10, 213

Dunkley, J. et al., arXiv:0803.0586

Eisenstein, D. \& Hu, W., 1998, ApJ, 496, 605

Feng, B., Wang, X. \& Zhang, X., 2005, Phys. Lett. B, 607, 35

Freedman, W., et al., 2001, ApJ, 553, 47

Hinshaw, G. et al., arXiv: 0803.0732

Huterer, D. \& Starkman, G., 2003, Phys. Rev. Lett., 90, 031301

Lewis, A. \& Bridle, S., 2002, Phys. Rev. D, 66, 103511

Linder, E., 2003, Phys. Rev. Lett., 90, 091301

Komatsu, E. et al., 2008a, arXiv:0803.0547

Komatsu, E., private communication, 2008b.

Nolta, M., et al., arXiv: 0803.0593

Peebles, P. J. E. \& Ratra, B., 1998, ApJ, 325, L17

Ratra, B. \& Peebles, P. J. E., 1998, Phys. Rev. D, 37, 3406

Riess, A., et al., 2007, ApJ, 659, 98

Spergel, D., et al., 2007, ApJS, 170, 377

Wang, Y., \& Tegmark, M., 2005, Phys. Rev. D, 71, 103513 
Wang, Y. \& Mukherjee, P., 2007, Phys. Rev. D76, 103533

Weller, J. \& Lewis, A., 2003, MNRAS, 346, 987

Wetterich, C., 1995, ApJ, 301, 321

Wetterich, C., 1998, Nucl. Phys. B, 302, 668

Wright, E., 2007, ApJ, 664, 633

Xia, J., Zhao, G., Feng, B., Li, H. \& Zhang, X., 2006, Phys. Rev. D, 73, 063521

Yeche, Ch., Ealet, A., Refregier, A., Tao, C., Tilquin, A., Virey, J., \& Yvon, D., 2006, Astron. Astrophys., 448, 831

Zhao, G., Xia, J., Li, M., Feng, B. \& Zhang, X., 2005, Phys. Rev. D, 72, 123515

Zhao, G., et al., 2007a, Phys. Lett. B, 648, 8

Zhao, G., Xia, J., Feng, B. \& Zhang, X., 2007b, Int. J. Mod. Phys. D, 16, 1229

Zhao, G., Huterer, D., \& Zhang, X., 2007c, arXiv:0712.2277 